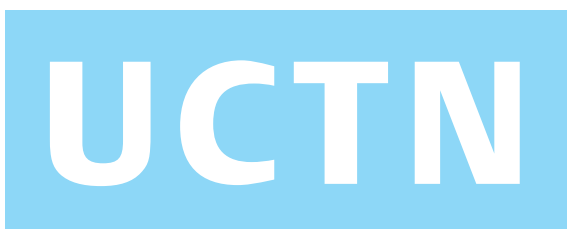

\title{
Giant diaphragmatic hernia with intrathoracic gastric volvulus
}

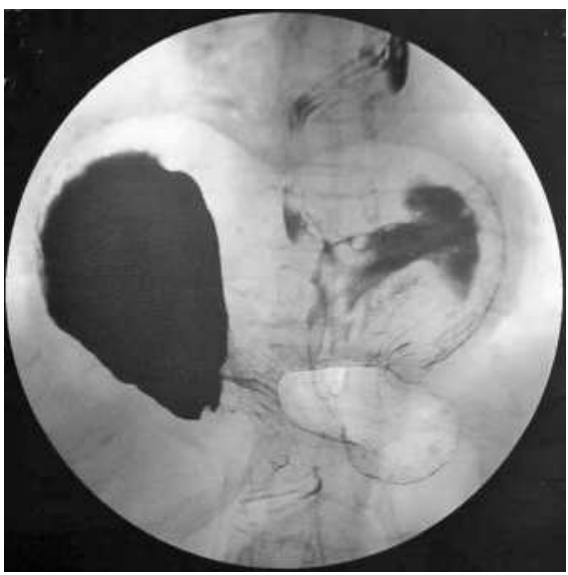

Figure 1 Upper gastrointestinal series showing a diaphragmatic hernia and an intrathoracic organoaxial gastric volvulus with gastric outlet obstruction.

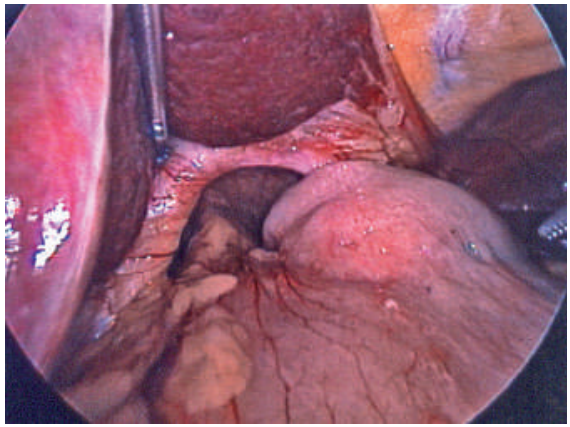

Figure 2 At laparoscopy a diaphragmatic defect, $7 \times 5 \mathrm{~cm}$ in diameter, was seen at the esophageal hiatus, with a well-developed hernial sac inside it after herniated organ reduction.

The traditional surgical management of gastric volvulus consists of laparotomy with gastric detorsion and fixation, and diaphragmatic hernia repair. We report here an 83-year-old patient whose gastric volvulus occurred through a giant diaphragmatic defect (Figure 1), who presented with a 1-week history of abdominal pain, distension, vomiting, and dysphagia. At surgery, the gastroesophageal junction, the fundus and distal half of the stomach, and the omentum were found in the thorax, and an intrathoracic organoaxial gastric volvulus through a hiatal hernia was diagnosed (Figure 2 ). We proceeded to laparoscopic herniated organ reduction, excision of the hernial sac, mo-

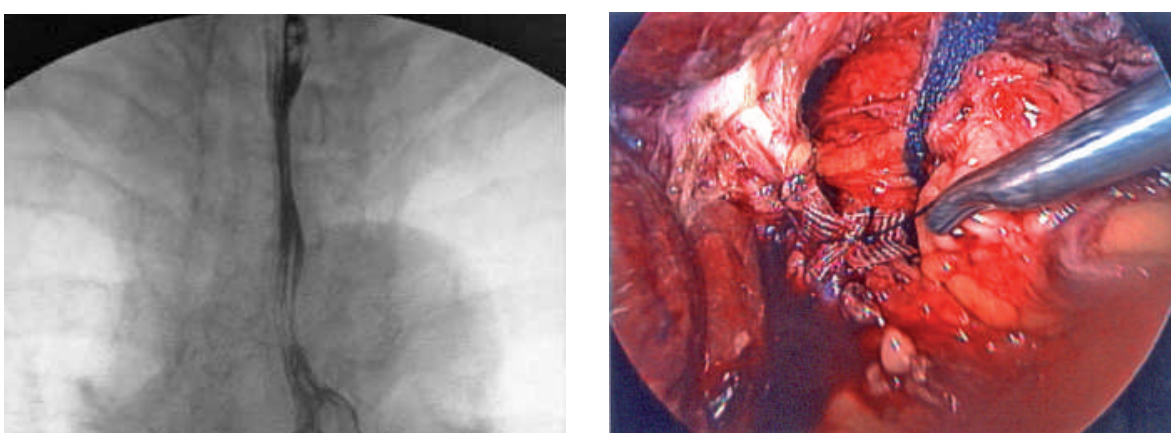

Figure 5 The crura were repaired posterior to the esophagus, with several interrupted nonabsorbable sutures and pieces of mesh used as pledgets for reinforcement.

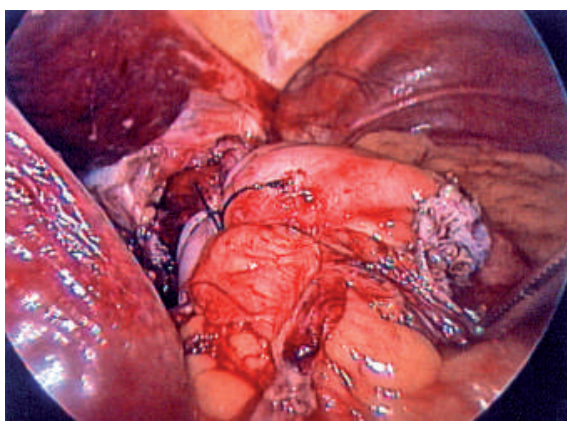

Figure 3 Hypaque study demonstrating adequate stomach position, good gastric emptying, and adequate detorsion and fixation of the stomach postoperatively.

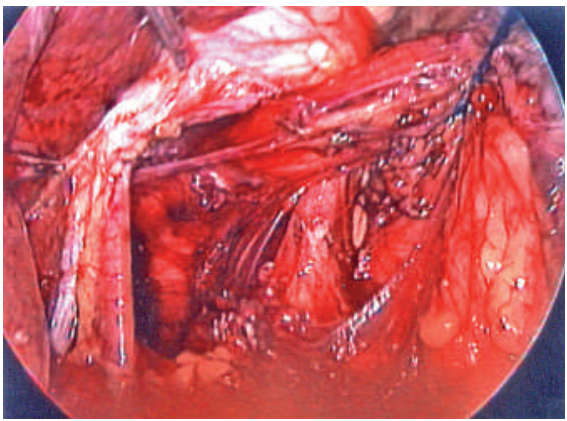

Figure 4 The inferior parts of the right and left crura were clearly exposed after the esophagus was pulled upward and to the left.

bilization of the fundus, crural repair, and a Nissen fundoplication and gastropexy. The patient was well and had no hernia recurrence at 1-year follow-up (Figure 3).

During these procedures, the first important step was to make sure we avoided entering the pleura and creating a tension
Figure 6 A standard Nissen fundoplication was performed, using a short, floppy, 2-cm fundic wrap, and stitch fixation of the fundic wrap to right crus.

pneumothorax while making the window behind the esophagus (Figure 4). We also chose to approximate tissues using stout, nonabsorable sutures, taking healthy bites into both sides of the crura posteriorly, and used pieces of mesh to reinforce all the crural repairs (Figure 5 ). This reduced the risk of ischemic necrosis and subsequent failure of the crural repair. In addition, this meant that we did not need to place prosthetic mesh across the hiatal defect and the complications associated with this were also avoided $[1,2]$. We thought that suturing the fundic wrap to would be effective for gastropexy because excessive mobility of the greater curvature of the stomach was restricted after fundoplication (Figure 6). The need for

DOI: $10.1055 / \mathrm{s}-2006-944793$ the right crus with a nonabsorbable stitch 
gastrostomy for gastropexy suggested in the study by Geha et al. [3] was obviated.

It is a long-held belief that ageing is associated with a gradual loss of cardiopulmonary reserve and that pneumoperitoneum is dangerous in the elderly [4]. Recently, Ballesta et al. [5] concluded that pulmonary complications might be reduced after a laparoscopic procedure compared with an open approach in elderly patients. Laparoscopic surgery would appear to be the ideal choice of treatment for the elderly because of its minimal invasiveness.

Endoscopy_UCTN_Code_CCL_1AB_2AC_3AC
C.-C. Chiu ${ }^{1}$, W. Wang ${ }^{2}$, P.-L. Wei ${ }^{3}$, W.-J. Lee ${ }^{2}$

${ }^{1}$ Department of Surgery, Chi Mei Medical Center, Liouying Campus, Liouying

Township, Tainan County, Taiwan

2 Department of Surgery, Min Sheng General Hospital, Taoyuan County, Taiwan

${ }^{3}$ Department of Surgery, Taipei Medical University Hospital, Taipei City,

Taiwan.

\section{References}

${ }^{1}$ Leeder PC, Smith G, Dehn TC. Laparoscopic management of large paraesophageal hiatal hernia. Surg Endosc 2003; 17: 1372 - 1375

2 Targarona EM, Bendahan G, Balague $C$ et al. Mesh in the hiatus: a controversial issue. Arch Surg 2004; 139: 1286-1296
${ }^{3}$ Geha AS, Massad MG, Snow NJ et al. A 32year experience in 100 patients with giant paraesophageal hernia: the case for abdominal approach and selective antireflux repair. Surgery 2000; 128: 623-630

${ }^{4}$ Weber DM. Laparoscopic surgery: an excellent approach in elderly patients. Arch Surg 2003; 138: 1083 - 1088

${ }^{5}$ Ballesta LC, Cid JA, Poves I et al. Laparoscopic surgery in the elderly patient. Surg Endosc 2003; 17: $333-337$

\section{Corresponding author}

\section{W.-J. Lee, M.D.}

Department of Surgery

Min Sheng General Hospital

168 Ching-Kuo Road

330 Taoyuan

Taiwan

Fax: $\quad+886-3-3469291$

E-mail: zaqwedcxs0399@sina.com.tw 